\title{
Comparação de Segmentos Angiograficamente Normais e com Estenose Luminal Significativa Avaliados pelo Ultrassom Intracoronário com Histologia Virtual: Análise Piloto
}

\author{
Gustavo Rique Morais¹, Breno de Alencar Araripe Falcão ${ }^{1}$, João Luiz de Alencar Araripe Falcão¹, \\ Rafael Cavalcante e Silva ${ }^{1}$, Expedito Eustáquio Ribeiro da Silva ${ }^{1}$, Pedro Alves Lemos ${ }^{1}$
}

\begin{abstract}
RESUMO
Introdução: A doença arterial coronária (DAC) é sabidamente difusa, podendo acometer múltiplos segmentos arteriais. No entanto, quando se analisa a angiografia coronária, é comum a concomitância, em um mesmo paciente, da presença de lesão em um segmento e da ausência de obstrução em outros. Por se tratar de um luminograma, a angiografia apresenta limitações para o diagnóstico da DAC. Neste estudo utilizou-se o ultrassom intracoronário com histologia virtual (USIC-HV) para avaliar a presença e a composição de placas ateroscleróticas em segmentos angiograficamente normais e com lesões em pacientes com DAC estabelecida. Métodos: No total, 17 pacientes com DAC obstrutiva encaminhados para realização de angioplastia foram submetidos a USIC-HV dos três grandes vasos epicárdicos. Após análise minuciosa da angiografia, foram selecionados 32 segmentos angiograficamente normais, os quais foram comparados a outros 19 segmentos coronários com estenose luminal $>70 \%$. Resultados: A carga de placa média em segmentos sem lesão angiográfica foi de 47,3 $15,1 \%$, enquanto em segmentos com estenose angiográfica foi de $76,2 \pm 8,8 \%(P<0,01)$. A composição da placa diferiu entre os segmentos. Placas nos segmentos angiograficamente normais apresentavam maior componente fibrolipídico $(17 \pm 11 \%$ vs. $11,4 \pm 12 \% ; \mathrm{P}=0,045)$ e menor componente necrótico $(13,1 \pm 13 \%$ vs. $20,2 \pm 12 \%$; $P=0,03)$. Conclusões: Nesta análise piloto, em pequeno grupo de pacientes portadores de DAC, observou-se que segmentos angiograficamente normais frequentemente apresentam placa aterosclerótica. A composição da placa em segmentos angiograficamente normais, entretanto, diferiu daquela em
\end{abstract}

\section{ABSTRACT}

Comparison of Angiographically Normal Segments and Segments with Significant Luminal Stenosis by Intracoronary Ultrasound and Virtual Histology: Preliminary Results

Background: Coronary artery disease (CAD) is known to be diffuse and can affect multiple arterial segments. However, findings of concomitant lesions in a single segment and no obstructions in other segments of the same patient are not unusual when assessing coronary angiographies. Because it is a luminogram, angiography has limitations for the diagnosis of CAD. In this study, intravascular ultrasound and virtual histology (IVUS-VH) were used to assess the presence and composition of atherosclerotic plaques in angiographically normal segments and in segments with lesions of patients with established CAD. Methods: A total of 17 patients with obstructive CAD referred for angioplasty were submitted to IVUS-VH of the three major epicardial coronary arteries. After careful angiographic analysis, 32 angiographically normal segments were selected and compared to 19 segments with luminal stenosis > 70\%. Results: Mean plaque burden in angiographically normal segments was $47.3 \pm 15.1 \%$, whereas in segments with angiographic stenosis it was $76.2 \pm 8.8 \%(\mathrm{P}<0.01)$. Plaque composition differed among segments. Plaques in angiographically normal segments had higher percentage of fibrofatty component $(17 \pm 11 \%$ vs. $11.4 \pm 12 \% ; P=0.045)$ and lower percentage of necrotic component $(13.1 \pm 13 \%$ vs. $20.2 \pm 12 \%$; $\mathrm{P}=0.03)$. Conclusions: In this pilot study, in a small group of patients with CAD, it was observed that angiographically normal

1 Instituto do Coração do Hospital das Clínicas da Faculdade de Medicina da Universidade de São Paulo (InCor/HCFMUSP) - São Paulo, SP, Brasil.

Correspondência: Gustavo Rique Morais. Rua São Gonçalo, 416/ 1.401 - Manaíra - João Pessoa, PB, Brasil - CEP 58038-330 E-mail: gustavorique@hotmail.com

Recebido em: 4/7/2011 • Aceito em: 30/8/2011 
segmentos com lesão, sugerindo a coexistência de estágios distintos do processo aterosclerótico em um mesmo paciente.

DESCRITORES: Placa aterosclerótica. Angiografia. Doença da artéria coronariana.

A doença arterial coronária (DAC) aflige grande número de pessoas em todo o mundo, de forma quase epidêmica em países desenvolvidos e em desenvolvimento. É a principal causa de morbidade e mortalidade, juntamente com as doenças cerebrovasculares. Segundo a Organização Mundial de Saúde (OMS), estima-se que, em 2020, 36 em cada 100 pessoas serão vítimas de infarto agudo do miocárdio (IAM), podendo chegar a alarmantes 25 milhões de mortes no ano.

Nos dias de hoje, o tratamento da DAC é largamente norteado pela presença ou ausência de obstrução luminal. Habitualmente, a conduta terapêutica adotada para indivíduos com coronariopatia obstrutiva inclui a administração de medicamentos anti-isquêmicos e a realização de tratamento mecânico por meio de métodos invasivos que propiciam o restabelecimento do fluxo coronário, tais como a angioplastia coronária e a cirurgia de revascularização miocárdica. Fica evidente, dessa maneira, que a angiografia coronária desempenha papel fundamental no direcionamento terapêutico do paciente com DAC.

Apesar de ser considerado padrão de referência para o diagnóstico da doença, o método apresenta algumas limitações importantes. A angiografia baseia-se no delineamento da anatomia da luz coronária. O diagnóstico de estenose luminal é realizado comparando-se um ponto de menor calibre com outro de calibre aparentemente normal, utilizado como referência. Em uma proporção de casos, a presença de placa(s) aterosclerótica(s) com distribuição difusa pode prejudicar a obtenção da obstrução luminal verdadeira pela angiografia coronária, em decorrência da ausência de segmentos com luz normal para comparação (isto é, sítios com maior acúmulo de placa podem ter seu verdadeiro grau de obstrução subestimado ao serem confrontados com outra região também afetada pela doença). Além de subestimar o grau de estenose em alguns casos, em outros, a doença coronária difusa pode per se gerar aumento da resistência ao fluxo, pela presença de longos segmentos afetados por placas ateroscleróticas. ${ }^{1}$ Em ambos os casos, o segmento patológico apresenta aspecto angiográfico normal, o que, na prática clínica, impede o reconhecimento do componente obstrutivo existente nesses pacientes.

Por outro lado, estudos anatomopatológicos e com ultrassom intravascular demonstraram que a presença segments often have atherosclerotic plaque. However, plaque composition was different in angiographically normal segments and those with angiographic stenosis, suggesting the coexistence of distinct stages of the atherosclerotic process in the same patient.

KEY-WORDS: Plaque, atherosclerotic. Angiography. Coronary artery disease.

de placa aterosclerótica não determina necessariamente que haja obstrução luminal significativa..$^{2-5}$ Frequentemente, o acúmulo de placa aterosclerótica é acompanhado pelo crescimento localizado das dimensões arteriais, o que permite o acomodamento da placa sem que haja redução do calibre da luz. ${ }^{2-5}$ Obviamente, esse fenômeno de remodelamento vascular impede a identificação da placa aterosclerótica pela angiografia coronária, uma vez que o lúmen vascular se apresenta normal. No entanto, diversos estudos têm demonstrado nas últimas décadas que mesmo placas ateroscleróticas sem obstrução significativa à angiografia têm potencial para desencadear complicações coronárias agudas, ${ }^{6-9}$ associadas a fenômenos aterotrombóticos locais. ${ }^{6,7}$

Em resumo, segmentos coronários sem obstrução luminal evidente à angiografia podem apresentar três situações distintas em pacientes com coronariopatia obstrutiva em outros segmentos: 1) ausência de placa aterosclerótica, 2) placa aterosclerótica sem obstrução luminal (isto é, com remodelamento focal adaptativo), e 3) placa aterosclerótica difusa com estenose subestimada pela angiografia (em decorrência da ausência de segmento de referência normal para comparação).

Neste estudo utilizou-se o ultrassom intracoronário com histologia virtual (USIC-HV) para avaliar a presença e a composição de placas ateroscleróticas em segmentos angiograficamente normais, comparando-os com segmentos com lesões em pacientes com DAC estabelecida.

\section{MÉTODOS}

Foram selecionados 17 pacientes não-consecutivos, que foram encaminhados ao laboratório de hemodinâmica para realização de angioplastia coronária com implante de stent, em uma ou mais lesões coronárias. O USIC foi utilizado para guiar o procedimento, bem como para obter imagens de segmentos coronários proximais de pelo menos uma artéria não submetida à intervenção. Todos os pacientes assinaram o Termo de Consentimento Livre e Esclarecido, aprovado pela Comissão de Ética do Hospital das Clínicas da Faculdade de Medicina da Universidade de São Paulo (São Paulo, SP, Brasil).

Foram excluídos pacientes que não tivessem pelo menos $20 \mathrm{~mm}$ proximais do vaso não-tratado pela intervenção percutânea avaliados pelo USIC ou que apresentassem imagens com qualidade inadequada. 
As imagens angiográficas foram obtidas em diferentes projeções, separadas por pelo menos 30 graus (mínimo de duas projeções para a coronária direita e de três projeções para a coronária esquerda). A análise da angiografia foi realizada por estimativa visual por dois cardiologistas intervencionistas independentes.

A avaliação pelo USIC consistiu da análise quantitativa volumétrica, como também da histologia virtual (Volcano Therapeutics Inc., Rancho Cordova, Estados Unidos). As imagens do USIC volumétrico e da histologia virtual foram adquiridas pelo mesmo cateter de ultrassom intravascular, simultaneamente, com o auxílio do pullback automático, na velocidade de $0,5 \mathrm{~mm} / \mathrm{segun}-$ do. Foram examinadas as porções proximais de pelo menos um vaso epicárdico sem lesão significativa à angiografia (idealmente os segmentos proximais dos três vasos epicárdicos deveriam ser analisados). Uma extensão mínima de $20 \mathrm{~mm}$ (a partir do óstio aortocoronário) foi analisada (idealmente $>40 \mathrm{~mm}$ ). Em todos os exames foi utilizada nitroglicerina intracoronária previamente à obtenção das imagens.

Os seguintes parâmetros do USIC volumétrico foram analisados off-line na estação Pie Medical BV (Maastricht, Holanda): extensão do segmento analisado, área média do vaso, área média da luz, área média da placa, e obstrução porcentual média da placa. Posteriormente foram avaliados, com o auxílio de software específico, os parâmetros de histologia virtual: conteúdo porcentual médio e área média de componentes de cálcio, fibroso, fibrolipídico e necrótico.

Após análise da angiografia, foram selecionados 32 segmentos angiograficamente normais. Foram excluídos segmentos com "irregularidades" luminais e todos os segmentos normais distavam pelo menos $10 \mathrm{~mm}$ de segmentos com quaisquer evidências angiográficas de lesão. Para comparação, foram também selecionados 19 segmentos com estenose luminal significativa à angiografia (> 70\%). Foram avaliadas as imagens de USIC-HV, sendo computada, para a análise final, a secção com maior carga de placa nos segmentos angiograficamente normais e a secção com menor área luminal nos segmentos com lesão angiográfica.

Utilizou-se o programa estatístico SPSS versão 17 para Windows. As variáveis categóricas foram apresentadas como números absolutos e porcentagens. As variáveis contínuas foram descritas como média \pm desvio padrão e comparadas com o teste $t$ de Student. Valor de $\mathrm{P}$ bicaudal $<0,05$ foi considerado significativo.

\section{RESULTADOS}

A média de idade dos pacientes foi de 60,5 \pm 11,1 anos, sendo $70 \%$ do sexo feminino, 23\% diabéticos e $41 \%$ com diagnóstico de síndrome coronária aguda. As características clínicas dos pacientes são mostradas na Tabela 1.
Nos segmentos angiograficamente normais, a área luminal foi de 9,51 $\pm 4,86 \mathrm{~mm}^{2}$, enquanto nos segmentos com estenose angiográfica foi de $2,8+0,71 \mathrm{~mm}^{2}$ $(\mathrm{P}<0,01)$ (Tabela 2). Na média, segmentos angiograficamente normais apresentavam carga de placa de $47,3 \pm 15,1 \%$, enquanto os segmentos com lesão angiográfica apresentavam carga de placa de 76,2 \pm $8,8 \%(P<0,01)$ (Figura). Entre os segmentos angiograficamente normais, apenas $3(9,3 \%)$ não apresentavam placa aterosclerótica ao USIC.

Na comparação dos segmentos angiograficamente normais com aqueles com lesão não foram encontradas diferenças em relação à composição da placa para o componente fibroso $(55 \pm 20 \%$ vs. $60,7 \pm 12 \%$; $\mathrm{P}=0,68)$ e o componente de cálcio $(5,5 \pm 6 \%$ vs. 7,7 $\pm 7 \% ; P=0,23)$. Os segmentos angiograficamente normais apresentaram maior componente fibrolipídico $(17 \pm 11 \%$ vs. 11,4 $\pm 12 \% ; P=0,045)$ e menor componente necrótico $(13,1 \pm 13 \%$ vs. $20,2 \pm 12 \%$; $P=0,03)$ (Tabela 3).

\section{DISCUSSÃO}

A angiografia coronária foi considerada, por muito tempo, padrão de referência para o diagnóstico de DAC. Entretanto, inúmeros estudos indicaram que a extensão da DAC é subestimada pela análise visual de segmentos angiograficamente "normais". Mintz et al. ${ }^{4}$ demonstraram, por meio da análise com USIC, que apenas $6,8 \%$ de 884 segmentos considerados normais pela angiografia apresentavam realmente ausência de placa aterosclerótica. A carga de placa média desses segmentos foi de $51 \pm 13 \%$. Nesse estudo, no entanto, foram analisados somente segmentos adjacentes às porções com lesão angiográfica. Estudos de anatomia patológica corroboram tal achado, demonstrando grande discrepância entre os achados angiográficos e os da patologia. Uma possível explicação seria a dilatação compensatória da parede arterial ao acúmulo da placa aterosclerótica, o chamado remodelamento positivo.

TABELA 1 Características clínicas

\begin{tabular}{lc} 
Variáveis & $\mathbf{n = 1 7}$ \\
\hline Idade, anos & $60,5 \pm 11,1$ \\
Sexo feminino, \% & 70 \\
Diabetes melito, \% & 23 \\
Hipertensão arterial, \% & 88 \\
Hipercolesterolemia, \% & 70 \\
História de tabagismo, \% & 59 \\
Quadro clínico, \% & \\
$\quad$ Angina estável & 59 \\
Síndrome coronária aguda & 41 \\
\hline $\mathrm{n}=$ número de pacientes. & \\
\hline
\end{tabular}


TABELA 2

Medidas ultrassonográficas em segmentos normais ou com lesão à angiografia

\begin{tabular}{lccc}
\hline & $\begin{array}{c}\text { Segmentos } \\
\text { angiograficamente } \\
\text { normais (32 segmentos) }\end{array}$ & $\begin{array}{c}\text { Segmentos com } \\
\text { lesão à angiografia } \\
\text { (19 segmentos) }\end{array}$ & Valor de P \\
\hline Área luminal, $\mathrm{mm}^{2}$ & $9,51 \pm 4,86$ & $2,8 \pm 0,71$ & $<0,01$ \\
Área da placa, $\mathrm{mm}^{2}$ & $8,85 \pm 4,16$ & $10,38 \pm 5,1$ & 0,25 \\
Área da lâmina elástica externa, $\mathrm{mm}^{2}$ & $18,4 \pm 7,9$ & $13,2 \pm 5,3$ & 0,02 \\
Carga de placa, \% & $47,3 \pm 15,1$ & $76,2 \pm 8,8$ & $<0,01$ \\
\hline
\end{tabular}

TABELA 3

Componentes da placa aterosclerótica em segmentos normais ou com lesão à angiografia

\begin{tabular}{lccc}
\hline $\begin{array}{l}\text { Componente da } \\
\text { placa aterosclerótica }\end{array}$ & $\begin{array}{c}\text { Segmentos } \\
\text { angiograficamente normais }\end{array}$ & $\begin{array}{c}\text { Segmentos com } \\
\text { lesão à angiografia }\end{array}$ & Valor de P \\
\hline Fibroso, \% & $55 \pm 20$ & $60,7 \pm 12$ & 0,68 \\
Fibrolipídico, \% & $17 \pm 11$ & $11,4 \pm 12$ & 0,045 \\
Necrótico, \% & $13,1 \pm 13$ & $20,2 \pm 12$ & 0,03 \\
Cálcio, \% & $5,5 \pm 6$ & $7,7 \pm 7$ & 0,23 \\
\hline
\end{tabular}

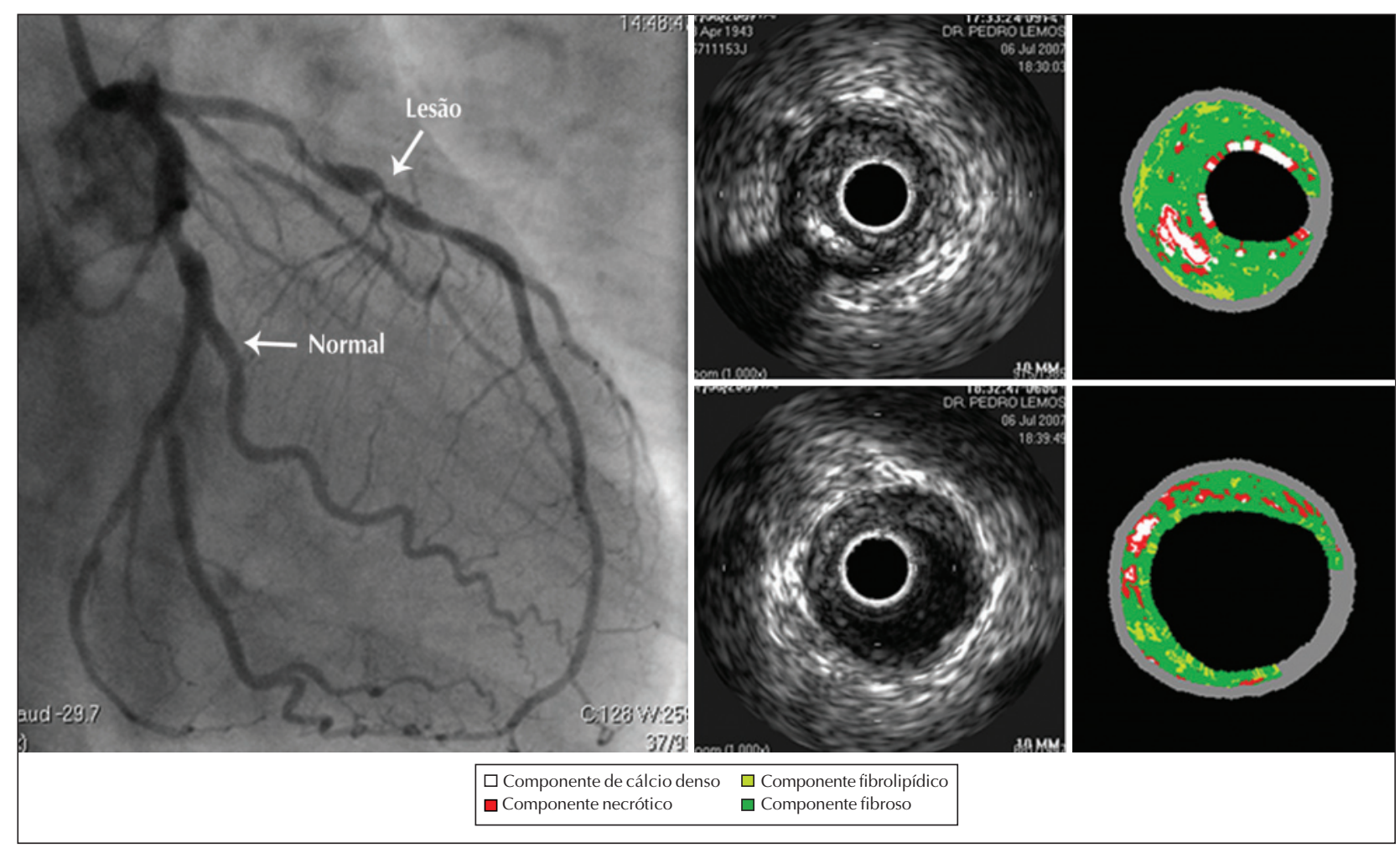

Figura - Comparação entre segmentos com e sem lesão à angiografia com ultrassom intracoronário com histologia virtual (USIC-VH) em um mesmo paciente.

Neste estudo foram analisados segmentos angiograficamente normais, em sítios distantes dos segmentos com lesão manifesta. Demonstrou-se que, mesmo na- queles segmentos, pode haver placa aterosclerótica, com carga de placa média de 47,3 $\pm 15,1 \%$. O presente estudo analisou também a composição da placa 
aterosclerótica por meio da histologia virtual. A composição da placa diferiu entre segmentos com estenose significativa submetidos a angioplastia coronária e segmentos considerados normais pela angiografia. Os segmentos com estenose apresentaram composição de placa com maior prevalência do componente core necrótico, enquanto os segmentos com angiografia normal apresentaram maior prevalência do componente fibrolipídico. Tal achado sugere que, apesar de difusamente presente ao longo da árvore coronária, é provável que o processo aterosclerótico apresente evolução e estágios distintos em diferentes segmentos arteriais.

\section{Limitações do estudo}

Algumas limitações devem ser consideradas na interpretação dos resultados deste estudo. Trata-se de casuística não-consecutiva, com número modesto de pacientes, em que vieses de seleção podem ter influenciado os achados. Este foi um estudo in vivo e uma comparação com dados histopatológicos é necessária para confirmar os achados do USIC-HV.

\section{CONCLUSÕES}

Nesta análise piloto em pequeno grupo de pacientes coronariopatas, observou-se a presença frequente de placa aterosclerótica ao USIC em segmentos angiograficamente normais. No entanto, a composição da placa nesses segmentos difere da composição em segmentos com lesão angiográfica, sugerindo estágios distintos no processo aterosclerótico.

\section{SUPORTE FINANCEIRO}

Este estudo teve o apoio da Fundação de Amparo à Pesquisa do Estado de São Paulo (FAPESP).

\section{CONFLITO DE INTERESSES}

Os autores declaram não haver conflito de interesses relacionado a este manuscrito.

\section{REFERÊNCIAS}

1. De Bruyne B, Hersbach F, Pijls NH, Bartunek J, Bech JW, Heyndrickx GR, et al. Abnormal epicardial coronary resistance in patients with diffuse atherosclerosis but "normal" coronary angiography. Circulation. 2001;104(20):2401-6.

2. Glagov S, Weisenberg E, Zarins CK, Stankunavicius R, Kolettis G). Compensatory enlargement of human atherosclerotic coronary arteries. N Engl J Med. 1987;316(22):1371-5.

3. Mintz GS, Kent KM, Pichard AD, Satler LF, Popma J, Leon $M B$. Contribution of inadequate arterial remodeling to the development of focal coronary artery stenoses: an intravascular ultrasound study. Circulation. 1997;95(7):1791-8.

4. Mintz GS, Painter JA, Pichard AD, Kent KM, Satler LF, Popma J, et al. Atherosclerosis in angiographically "normal" coronary artery reference segments: an intravascular ultrasound study with clinical correlations. J Am Coll Cardiol. 1995;25(7):1479-85.

5. Briguori C, Tobis J, Nishida T, Vaghetti M, Albiero R, Di Mario C, et al. Discrepancy between angiography and intravascular ultrasound when analysing small coronary arteries. Eur Heart J. 2002;23(3):247-54.

6. Naghavi M, Libby P, Falk E, Casscells SW, Litovsky S, Rumberger J, et al. From vulnerable plaque to vulnerable patient: a call for new definitions and risk assessment strategies: Part II. Circulation. 2003;108(15):1772-8.

7. Naghavi M, Libby P, Falk E, Casscells SW, Litovsky S, Rumberger J, et al. From vulnerable plaque to vulnerable patient: a call for new definitions and risk assessment strategies: Part I. Circulation. 2003;108(14):1664-72.

8. Ambrose JA, Tannenbaum MA, Alexopoulos D, HjemdahlMonsen CE, Leavy J, Weiss M, et al. Angiographic progression of coronary artery disease and the development of myocardial infarction. J Am Coll Cardiol. 1988;12(1):56-62.

9. Smith SC Jr. Risk-reduction therapy: the challenge to change. Presented at the $68^{\text {th }}$ Scientific Sessions of the American Heart Association (AHA); 1995 Nov 13; Anaheim, California. Circulation. 1996;93(12):2205-11. 